University of Nebraska - Lincoln

DigitalCommons@University of Nebraska - Lincoln

20th \& 21st Century French and Francophone Modern Languages and Literatures, Department Studies International Colloquium

\title{
La terre natale : à la source de l'écriture, chez Sidonie Gabrielle Colette
}

Michèle Druon

Follow this and additional works at: https://digitalcommons.unl.edu/ffsc2020

Part of the Comparative Literature Commons, French and Francophone Literature Commons, and the Other French and Francophone Language and Literature Commons

This Presentation is brought to you for free and open access by the Modern Languages and Literatures, Department of at DigitalCommons@University of Nebraska - Lincoln. It has been accepted for inclusion in 20th \& 21st Century French and Francophone Studies International Colloquium by an authorized administrator of DigitalCommons@University of Nebraska - Lincoln. 


\title{
La terre natale : à la source de l'écriture, chez Sidonie Gabrielle Colette
}

\author{
par Dr. Michèle Druon
}

\begin{abstract}
Mais le pays natal est moins une étendue qu'une matière ; c'est un granit ou une terre, un vent ou une sécheresse, une eau ou une lumière ; c'est par lui que notre rêve prend sa juste substance ; c'est à lui que nous demandons notre couleur fondamentale.

Gaston Bachelard, L'Eau et les Rêves.
\end{abstract}

Tout au long de son œuvre, Colette a cherché à ressusciter dans ses textes le « paradis terrestre » de son pays natal, la Bourgogne, et le rêve profond qui lui est attaché. Déjà évoquée dans les scènes campagnardes du premier Claudine (Claudine à l'Ecole,1900), la terre natale réapparait dans de courtes nouvelles comme Les Vrilles de La Vigne (1908) et revient au premier plan dans des textes consacrés à la maison natale de Saint-Sauveur en Puisaye et à la mère de Colette, Sido : La Maison de Claudine (1922), La Naissance du Jour (1928), et Sido (1930). Foyer d'un complexe de souvenirs, sensations, désirs et émotions, la terre de l'enfance génère dans l'écriture de Colette une chaîne d'images affectivement et symboliquement connectées: images de campagne et de forêts, de sources pures, de renaissance et de début du monde, et d'un « domaine nourricier » étroitement associé à la figure de sa mère. Dans ces images se dessine un rêve édénique d'harmonie et de fusion avec la nature, qui se prolonge et se ramifie dans les textes plus tardifs comme Prisons et Paradis (1932-49), Flore et Pomone (1944), Belles Saisons (1945), et Paradis Terrestre (1953), entièrement consacrés au monde animal et végétal. A travers ce trajet de retour symbolique (1) au pays natal, retour aux sources et « aux eaux vives de l'enfance », Colette déploie une écriture éblouissante, tout entière attachée à « faire parler » la terre.

L'évocation du pays natal, que Colette a quitté à l'âge de 18 ans, est d'emblée marquée par une profonde nostalgie pour son environnement naturel, milieu « délicieux et apaisant », porteur d'une plénitude à la fois sensuelle et spirituelle :

J'appartiens à un pays que j'ai quitté. Tu ne peux empêcher qu'à cette heure, si épanouie au soleil, sous la chevelure embaumée des forêts, l'herbe profonde noie le pied des arbres d'un vert délicieux et apaisant, dont mon âme a soif. Flore et Pomone, dans Gigi (1944) (2)

Cette « soif » des forêts de la terre natale inclut aussi ses sources (3), longuement et lyriquement célébrées dans des passages riches de portée symbolique; sources secrètes, cachées dans les bois, 
«sources perdues sitôt que nées », sources que Colette « révérait », et dont elle rêve d'emporter une « gorgée imaginaire » (*c'est moi qui souligne dans les passages suivants) :

Je revenais à la cloche de la première messe. Mais pas avant d'avoir mangé mon saoul, pas avant d'avoir dans les bois, décrit un grand circuit de chien qui chasse seul, et goûté l'eau de deux sources perdues, que je révérais. L'une se haussait hors de la terre par une convulsion cristalline, une sorte de sanglot, et traçait elle-même son lit sableux. Elle se décourageait aussitôt née et replongeait sous la terre. L'autre source, presque invisible, froissait l'herbe comme un serpent, s'étalait secrète, au centre d'un pré où des narcisses, fleuris en ronde, attestaient seuls sa présence. La première avait goût de feuille de chêne, la seconde de fer et de tige de jacinthe... Rien qu'à parler d'elles je souhaite que leur saveur m'emplisse la bouche au moment de tout finir, et que j'emporte, avec moi, cette gorgée imaginaire. Sido

Un jardin sans source ne murmure pas assez et mes regrets ne se détachent pas encore des eaux vives de mon enfance, surgies à petit flot de ma terre natale perdues sitôt que nées, comme du pâtre, des chemineaux, des chiens chasseurs, du renard et de l'oiseau. Une autre était dans un bois et l'automne la couvrait de feuilles mortes ; une dans un pré, sous l'herbe et si parfaitement ronde qu'une couronne de narcisses blancs, aussi ronde qu'elle-même, décelait seule sa place au printemps. Une coulait en musique d'une berge de route ; une était un joyau un peu bleu, tremblant dans une cuve de pierres grossièrement assemblées et des crevettes d'eau douce nageaient dans son ciel renversé. On m'assure que celle-ci est toujours aussi pure, mais qu'elle sautille, avec un vain effort de cristal, entre quatre parois de ciment, cadeau de la prévoyance humaine et je n'ai de goût que pour les sources sauvages, gardées par l'œil ouvert des myosotis et des candamines, par la grande salamandre tâchée comme un cheval pie. " Flore et Pomone

Associées à des images de plénitude (rondeur, couronne, douceur), de pureté et de de transparence (blancheur, cristal), ces sources se présentent comme un lieu idyllique, paradisiaque (« ciel renversé»), lieu de bonheur où se tiennent ensemble, en harmonie, tous les éléments de la terre : l'aquatique (les sources) le minéral (les pierres, le cristal, joyau); le végétal (l'l'herbe, les narcisses, les myosotis),, l'animal (les crevettes, la salamandre, le cheval pie).

Paradis terrestre, la terre de l'enfance est aussi celle d'un autre lieu de ravissement : le début du jour. Dans un passage qui semble faire écho à « L'aube», d'Arthur Rimbaud (« J'ai marché, réveillant les haleines vives et tièdes, et les pierreries regardèrent, et les ailes levèrent sans bruit »), le début du jour est chez Colette l'heure magique du premier réveil de la terre, d'une première « éclosion », d'une naissance du monde qui la plongeait, enfant, dans « un état de grâce indicible $»$ :

... J'allais seule, ce pays mal pensant était sans dangers. $C^{\prime}$ est sur ce chemin, c'est à cette heure que je prenais conscience de mon prix, d'un état de grâce indicible et de ma connivence avec le premier souffle accouru, le premier oiseau, le soleil encore ovale, déformé par son éclosion... Sido 
Etat d'éclosion toujours renouvelé, état de renaissance de la terre, l'aube est aussi - de manière cruciale - liée dans l'imaginaire de Colette à la figure de sa mère, Sido, comme en témoigne le titre du roman qu'elle lui a dédié : La Naissance du Jour. La figure de Sido est en effet indissociable chez Colette de ce que représente pour elle la terre natale. Influence centrale dans la vie de l'écrivaine, à la fois modèle et source d'inspiration, Sido est presque toujours évoquée dans ses textes en connexion avec le monde naturel : "parée d'enfants, de fleurs et d'animaux comme un domaine nourricier » (La Maison de Claudine, p. 60) ; elle est « inséparable » du domaine végétal et animal qui l'entoure: «Comme si je les découvrais ensemble, je saluai, inséparables, ma mère, le jardin et la ronde des bêtes.. (Ibid. p. 51).

Nouvelle Pomone, Sido est aussi celle qui initia Colette enfant aux mystères des plantes, et lui transmit pour elles son inlassable émerveillement :

Puissé-je n'oublier jamais que je suis la fille d'une telle femme qui penchait, tremblante, toutes ses rides éblouies entres les sabres d'un cactus sur une promesse de fleur, une telle femme qui ne cessa elle-même d'éclore, infatigablement, pendant trois quarts de siècle... a Naissance du Jour, p 6-7

A l'image des plantes qu'elle révère, cette éclosion toujours renouvelée de Sido semble naître de son contact avec la terre :

« où [Sido] semblait se sustenter de toute sève, et reprendre vie chaque fois chaque fois qu'en se baissant elle en touchait la terre » (Les Vrilles de la Vigne, p. 42)

Le lieu de ce contact avec la terre, de cette perpétuelle renaissance pour Sido, c'est son jardin. «Domaine nourricier» par excellence - car c'est un potager et un verger - ce jardin occupe une place importante dans l'enfance et les réminiscences de Colette :

Le Jardin-du-Haut commandait un Jardin-du-Bas, potager resserré et chaud, consacré à l'aubergine et au piment, où l'odeur du feuillage de la tomate se mêlait, en juillet, au parfum de l'abricot mûri sur espaliers. [...] La Maison de Claudine, p. 8

« Potager resserré et chaud » comme le cocon maternel, le jardin de Sido fait aussi fonction de microcosme de la campagne et de la terre qui l'entoure, et ouvre pour l'enfant sur une découverte sensorielle et sensuelle du monde naturel, métonymiquement induite dans ce passage par le contact avec la « robe de jardinière » de sa mère :

La robe de toile que je presse de ma joue sent le gros savon, la cire dont on lustre les fers à repasser, et la violette. Si je m'écarte un peu de cette fraîche robe de jardinière, ma tête plonge tout de suite dans une zone de parfum qui nous baigne comme une onde sans plis : le tabac blanc ouvre à la nuit ses tubes étroits de parfum et ses corolles en étoile. Un rayon, en touchant le noyer, 
l'éveille : il clapote, remué jusqu'aux basses branches par une mince rame de lune. Le vent superpose, à l'odeur du tabac blanc, l'odeur amère et froide des petites noix véreuses qui choient sur le gazon. Ibid. p 24

Cette « plongée » des sens dans le monde végétal décrit dans ce passage un double mouvement dans lequel le « je » de la narratrice s'efface à mesure que son environnement s'anime, " s'éveille » et devient agissant («touchant», « clapote », « superpose »....). La dissolution du moi dans un monde odorant, coloré, tactile provoque ici une euphorie et une plénitude (« une onde sans plis ») manifeste de la sensualité toujours intense et multiforme de Colette. Cette puissante sensualité lui permet de sentir et de ressentir très finement le monde qui l'entoure :

Moi, c'est mon corps qui pense. Il est plus intelligent que mon cerveau. Il ressent plus finement, plus complètement que mon cerveau. [...] Toute ma peau a une âme. La Retraite Sentimentale (1907)

Cette relation physique à son environnement animal et végétal se double chez Colette d'une profonde empathie, exprimée depuis l'enfance par un désir d'identification et de fusion avec le monde observé. Elle cherche à s'identifier à ce qu'elle observe pour mieux le comprendre « de l'intérieur ». Cette recherche d'identification physique et affective à des formes d'existence nonhumaines, ce désir d'un passage «transhumain » vers la nature, apparaît non seulement dans les représentations de l'animal chez Colette, mais aussi dans ses descriptions des plantes. On y observe le même double mouvement de disparition du « je » narrateur humain et son remplacement, ou sa métamorphose, en un « je » animal ou végétal (4).

Pour Julia Kristeva ((Le génie féminin, Tome III, Colette), le génie de Colette réside précisément dans « son corps métamorphique » qui lui permet de sortir de ses propres limites, de s'identifier physiquement à ce qui l'entoure, et d'être ainsi « poreuse » au monde. Elle analyse cette capacité en termes psychanalytiques comme « osmose totale » avec un « refoulé archaïque » :

Je vois dans ce mystère qui reste toutefois entier, la preuve la plus éloquente de son «corps métamorphique» $[\ldots](5)$

Colette se révèle capable d'une osmose totale avec le refoulé le plus archaïque, avec le prépsychique qui habite nos pulsions et nos sensibilités sous la frêle pellicule des mots qui le recouvrent, mais avec lequel son écriture a le génie de garder le contact afin de le réhabiliter et le transmettre.

Comme le souligne ici Kristeva, le génie de Colette réside aussi dans son écriture, qui réussit à « transmettre » ce rêve d'osmose à travers un langage qui lui est unique : 
Colette a trouvé un langage pour dire une étrange osmose entre ses sensations, ses désirs et ses angoisses $\{. .$.$\} et l'infini du monde (...) Ibid. p. 14$

[Son écriture] est une interpénétration de la langue et du monde, du style et de la chair, qui lui révèlent l'univers et les corps comme une « arabesque ». Ibid. . 15

Colette translate sa relation physique avec la terre, et l'univers de sensations et d'émotions qu'elle englobe, dans une écriture qui cherche à lui donner un langage. Car au cœur du rêve de paradis terrestre attaché au pays natal gît aussi chez Colette le désir, et la quête, de communiquer avec la nature, de comprendre son langage - de faire parler la terre. Dans le sillage de Sido dont elle se veut l'héritière, Colette cherche cet « alphabet nouveau », ou très ancien, qui pourrait être « le langage universel» de la nature et qui pourrait « dire tout ce qui n'a pas de nom dans le langage humain ». Cette recherche traverse toute l'écriture de Colette, et prend forme dans un somptueux réseau de métaphores qui, à l'instar des synesthésies baudelairiennes, invente de multiples correspondances entre les différents éléments de la nature :

[...] le muguet est une maigre fleurette à campanules ronds d'un blanc vert. Elle se hausse audessus des feuilles sèches, à l'heure de l'année où choient les premières pluies chaleureuses, gouttes lourdes qui entraînent, délient les arabesques simples échappées au bec du merle et les premières notes, d'une sphéricité lumineuse, jaillies des premiers rossignols... Je tâte timidement, j'invente un rapport indicible entre la goutte laiteuse des muguets, le pleur de pluie tiède, la bulle cristalline qui monte du crapaud ... Prisons et paradis

C'est la métaphore (5) ici, qui réinvente et transfigure le monde, non plus ressenti comme séparé mais relié dans tous ses éléments en un "rapport indicible » et harmonieux; par les correspondances et glissements de ses métaphores, Colette retisse le monde en une tapisserie vivante dont tous les éléments sont fluides et interconnectés, et qui peuvent aussi s'échanger et s'interpénétrer, dans un processus où s'effacent les séparations habituelles entre le végétal, l'animal et l'humain :

Ô violettes de mon enfance ! Vous montez devant moi, toutes, vous treillagez le ciel laiteux d'avril, et la palpitation de vos petits visages innombrables m'enivre... Les Vrilles de la Vigne.

Monstre à la langue poilue, c'est toi, suave iris ? Quelle maléfique grimace tord la lèvre de la rose à son réveil ! Vingt cornes de diable coiffent le bleuet, l'oeillet. Le pois grimpant darde une tête de python et la germination d'une poignée de lentilles mobilise une ruée d'hydres... Sido

Les fleurs peuvent avoir ici un corps humain ( "visages» des violettes, « langue » des iris, « lèvre » des roses) ou animal (« une tête de python »), et être douées du même « sexe exigeant », du même appétit de vivre, et de la même « férocité » : 
Sous nos yeux, le mimosa-sensitive, pour tromper l'agresseur, plie tous ses coudes de ramilles, abat ses aisselles de feuilles et ne livre qu'une dépouille évanouie. Un à un tombent les secrets des plantes, leurs défenses féeriques. Leurs pièges jouent à nu et dévoilent l'instinct carnassier, le goût du meurtre. Les bords vernissés, arrondis en lèvre, d'un calice cillé sont mortels. Une autre fleur referme sur l'insecte des herses entrecroisées de poils inflexibles... Eh ! quoi, elles sont méchantes, elles aussi ? Elles ont un sexe exigeant, une férocité, une fantaisie personnelles? Sido

Dans l'écriture de Colette, le monde humain, animal et végétal sont perçus et représentés comme catégories partagées de la nature. Cette représentation anticipe de manière frappante la perception écologique contemporaine de l'identité profonde du vivant (Bruno Latour). Elle illustre aussi la merveilleuse alchimie par laquelle l'écriture de Colette transfigure le monde, efface les séparations entre l'humain et l'animal, entre l'animal et le végétal, entre le sujet et l'objet, et dans cette symbiose magique, ressuscite enfin le paradis perdu de la terre natale.

\section{$\underline{\text { Notes : }}$}

1. Dans la Naissance du Jour, Colette décrit ainsi ce "retour à l'origine »: Tout est ressemblant aux premières années de ma vie, et je reconnais peu à peu, au rétrécissement du domaine rural, aux chats, à la chienne vieillie, à l'émerveillement, à une sérénité dont e sens de loin le souffle - (...) - je reconnais le chemin du retour ". ( $p$ 11)

2.Voir Colette, CEuvres, Tome I, II, III \& IV, Ed. La Pléiade, Paris (1984-2001)

3. Gaston Bachelard révèle une profonde affinité avec Colette dans sa fascination pour les sources de son pays natal (La Champagne):

"C'est près de l'eau et de ses fleurs que j'ai le mieux compris que la rêverie est un univers en émanation, un souffle odorant qui sort des choses par l'intermédiaire d'un rêveur. Si je veux étudier la vie des images de l'eau, il me faut donc rendre leur rôle dominant à la rivière et aux sources de mon pays.. [...]

En rêvant près de la rivière, j'ai voué mon imagination à l'eau, à l'eau verte et claire, à l'eau qui verdit les prés. . Je ne puis m'asseoir près d'un ruisseau sans tomber dans une rêverie profonde, sans revoir mon bonheur... Il n'est pas nécessaire que ce soit le ruisseau de chez nous, l'eau de chez nous. L'eau anonyme sait tous mes secrets. Le même souvenir sort de toutes les fontaines. "

4. Cette métamorphose peut prendre différentes formes et opérer à différents degrés - ainsi dans les textes de jeunesse comme Dialogues de Bêtes (1905) et Vrilles de la Vigne (1908), les animaux et les plantes servent essentiellement de support allégorique à la transposition de situations humaines et personnelles.

5. Julia Kristeva, Colette: Un Génie Féminin, Tome III, Fayard, 2002, p 40

6. Comme chez Paul Ricoeur, la métaphore chez Colette permet « l'éclatement du langage vers l'autre que lui-même : ce que j’appelle son ouverture ». (La Métaphore Vive, 1975) 